\title{
COLITIS ASOCIADA CON EL USO DE ANTIBIOTICOS
}

\author{
V. R. DOWELL, JR."
}

\begin{abstract}
La colitis seudomembranosa es una enfermedad diarreica relativamente poco común, y en ocasiones severa, asociada recientemente con la administración de algunos antibióticos de amplio espectro. Como agente causal se ha identificado una especie de clostridium esporulado, que hasta la fecha no había sido reconocido como patógeno para el humano. No obstante que este microorganismo es sensible a los antibióticos más comunes en uso, en sus esporos estaría la clave de la enfermedad.
\end{abstract}

La colitis seudomembranosa, caracterizada por la producción de placas inflamatorias y de seudomembranas en la mucosa del colon y por la diarrea severa que les acompaña, fue considerada desde tiempo atrás como una entidad clínica definida y clasificada como secuela ocasional de la cirugía del tracto intestinal, antes de la introducción de los antibióticos. Sin embargo, a partir de los años cincuenta se le asoció con la terapia por estos fármacos, relacionándose los primeros casos con el uso de derivados de la tetraciclina; hacia finales de la década se describió la colitis seudomembranosa e incluso el megacolon tóxico, en pacientes que estaban recibiendo algunos de estos antibióticos. Por la década de los sesenta, la ocurrencia de casos de colitis en pacientes que recibían neomicina en el preoperatorio de cirugía intestinal, inició un debate de consideración sobre el uso de la neomicina para estos propósitos. En 1963, Altemeier, Humel y Hill, en la Universidad de Cincinnati, comunicaron el aislamiento de una cepa de Staphylococcus aureus (fagotipo UC1 B), resistente a los antibióticos, en un alto porcentaje de pacientes quirúrgicos del Hospital General de

\footnotetext{
* Traducido del original, "Antibiotic-Associated Co litis. Hospital Practice: April, 1979: 75-80". Autori zado personalmente por su autor, Dr. V.R. Dowell, Jefe de la Sección de Enterobacteriología, de la División de Bacteriología del Centro para el Control de Enfermedades ) CDC), de Atlanta USA.
}

Cincinnati que desarrollaron colitis seudomembranosa y fue considerado como un microorganismo endémico en el hospital. La incidencia del gérmen declinó posteriormente así como la de los estafilococos resistentes a los antibióticos prevelentes por entonces en varios hospitales. Los cirujanos continuaron empleando la neomicina en la preparación del tracto intestinal antes de la cirugía, asociandola con algún otro agente, como la eritromicina o la bacitracina, para prevenir el crecimiento de gérmenes gram positivos.

Con la disminución del problema del estafilococo se presentó una reducción de los casos de colitis severa ligada con los antibióticos, aún cuando continuaron apareciendo en forma esporádica. En los comienzos de 1973 se describieron casos de colitis seudomembranosa asociados con el uso de dos antibióticos íntimamente relacionados, clindamicina y lincomicina, siendo llamativo el hecho de su aparición en brotes. Numerosos casos ocurrieron en un hospital, en un corto período de tiempo, en tanto que en otros hospitales de la misma área geográfica, donde se empleaban los mismos antibióticos, no se presentó ninguno. Aún cuando relacionado más frecuentemente con la terapia por clindamicina, la colitis seudomembranosa se presentaba también en pacientes que habían recibido penicilinas semisintéticas, tetraciclina, cloranfenicol u otros antibióticos de uso común. 
La variedad de síntomas en la diarrea, el dolor abdominal, la fiebre, la leucocitosis y en algunos casos el megacolon, hacen difícil el diagnóstico, particularmente en pacientes que han sido sometidos a cirugía intestinal o que han padecido cáncer colorectal. Por los estudios de F.J. Tedesco en la Universidad de Washington, San Louis, y los de otros autores, se estableció que las pruebas diagnósticas más confiables eran el examen proctoscópico, el estudio radiológico del colon con bario de contraste y la biopsia. La proctoscopia revela en la superficie de la mucosa las placas características de esta entidad, que también pueden ser apreciadas por el contraste con bario.

A pesar de que la terapéutica por antibióticos aparecía como el común denominador de los casos informados de colitis seudomembranosa, no se había establecido ninguna causa de manera concluyente. La condición había sido atribuida a un fenómeno de Shwartzman localizado, a toxicidad de las drogas, a isquemía entérica, a perturbaciones de la síntesis protéica en el tracto intestinal, a alteraciones en la flora bacteriana intestinal que permitían el predominio de cepas resistentes a los antibióticos, a organismos productores de toxinas o a infección viral de la mucosa del colon.

Ante la disyuntiva, varios grupos de investigadores comenzaron a estudiar intensamente el problema. La incognita principal era, si la enfermedad se presentaba como un efecto directo de la acción antibiótica sobre el tracto entérico, o más bien, como un efecto indirecto por la modificación de la flora intestinal que permitía la proliferación de organismos patógenos. Con este fin se desarrollaron varios modelos animales, ya que desde los primeros días de la era de la penicilina, se había visto que la administración de altas dosis de este antibiótico por vía intraperitoneal, producía en el cobayo una enfermedad fatal semejante a la colitis. Una respuesta similar se había producido en los hamsters por la administración de clindamicina o de lincomicina.

El examen del contenido cecal de los animales tratados mostró cambios en el equilibrio normal que existe entre las floras gram-positiva y gram-negativa. Estudios concomitantes sobre la bacteriología de las muestras fecales de pacientes con colitis seudomembranosa, revelaron una importante disminución en la flora anaeróbica, pero no permitieron implicar organismo alguno en particular.

El primer avance lo logró el grupo de H.E. Larson en Inglaterra al hallar una toxina en las heces de un paciente que, habiendo recibido fenoximitil penicilina por vía oral, desarrolló una colitis seudomembranosa. Al intentar el aislamiento de un virus que pudiera estar implicado como agente etiológico, Larson y sus colaboradores inocularon materias fecales del paciente en cultivos tisulares y observaron un efecto citopático sobre los fibroblastos, los que normalmente alargados, aparecían redondeados y ocasionalmente destruídos. Los mismos autores no encontraron evidencias de toxina en los especímenes fecales provenientes de pacientes con diarrea de otra etiología, específicamente salmonelosis o colitis ulcerativa. La relación entre la colitis asociada con los antibióticos y una toxina fue pronto confirmada por F.E. Fekety y su grupo en la Universidad de Michigan. Los filtrados de las heces de dos pacientes que desarrollaron la enfermedad luego de ser tratados con antibióticos, produjeron en cultivos de fibroblastos humanos los mismos efectos citopáticos descritos y los hamsters inoculados por vía intraperitoneal con estos filtrados, desarrollaron un sindrome tóxico muriendo a las 48 horas. La inoculación del filtrado de heces de los pacientes en la piel del conejo demostró la existencia de un factor de permeabilidad vascular (edema local, induración y hemorragia); este factor pudo ser inactivado por calentamiento del filtrado y neutralizado con antitoxina polivalente de la gangrena gaseosa. La antitoxina empleada fue una mezcla de las antitoxinas de Clostridium perfrigens, C. septicum, C.novyi, C. histolyticum y C. sordellii. Unicamente la antitoxina de $C$. sordellii, dio una reutralización específica para la toxina.

Uno de estos pacientes fue tratado por diez días con vancomicina ( $500 \mathrm{mg} /$ día) por vía oral, recuperándose. A los dos días de comenzado el tratamiento el filtrado de las 
heces dejó de ser tóxico para el hamster y para los cultivos de fibroblastos humanos y el factor de permeabilidad vascular desapareció. La toxina fue igualmente identificada por J.G. Bartlett, S.L. Gorbach y A.B. Onderdonk en el Hospital de Veteranos de Boston, quienes la descubrieron también cuando intentaban aislar virus de las heces de pacientes con colitis seudomembranosa.

El efecto citopático sobre los cultivos tisulares y la incapacidad de transferir este efecto de cultivo a cultivo, sugirió la implicación de una toxina más bien que de un virus. Los mismos autores habían observado un sobrecrecimiento de un clostridium, C. difficile, en el tracto intestinal de hamsters con un cuadro de enterocolitis inducido por clindamicina; este organismo se aisló de las heces de tres pacientes con diarrea asociada a la clindamicina. El filtrado de las heces, desprovisto de células, produjo citotoxicidad en los cultivos tisulares y enterocolitis en el hamster y la inyección intracecal de los gérmenes vivos provocó también un cuadro de enterocolitis. Tanto la acción citotóxica como la enterocilitis fueron neutralizadas por una antitoxina polivalente de la gangrena gaseosa.

Posteriormente, el grupo de Bartlett investigó en las muestras fecales de 189 individuos la presencia de la toxina citopática. 65 de éllos eran controles sanos; 10 pacientes presentaban una colitis ulcerativa y 24 niños tenían una colitis necrotizante neonatal; cuadros asociados con el uso de los antibióticos se presentaron en 47 pacientes con diarrea, 16 con una colitis "no específica" y 27 con una colitis seudomembranosa.

Por otra parte, se practicaron cultivos de las heces de 38 de los pacientes con colitis o diarrea asociadas con los antibióticos, recuperando el C. difficile en seis de ocho pacientes que presentaron pruebas de toxicidad positiva en los cultivos tisulares, y en cinco de treinta cuya prueba había sido negativa; tan solo de una muestra se aisló $C$. sordellii. También se probaron en cultivos tisulares de diferentes especies de clostridia y solamente con el C. difficile se demostró la toxina citopática. Todas las cepas de $C$ difficilefueron positivas y en todas, la acción citotóxica fue neutralizada por la antitoxina de C. sordellii. Los resultados de Bartlett fueron concluyentes para establecer que la colitis asociada con los antibióticos era causada por una toxina elaborada por el C. difficile.

C. difficile es un tipo de clostridium, esporulado y anaerobio obligado. Fue identificado por primera vez por I.C. Hall y E. O'Toole en 1935, quienes lo clasificaron como Bacillus difficilis por la dificultad que tuvieron para cultivarlo. Ellos lo informaron como un integrante normal de la flora intestinal del niño, aún cuando patógeno para un buen número de animales de laboratorio, incluidos los cobayos, las ratas, los hamsters y los conejos. Varios años después otros hallazgos fueron confirmados por M. L. Snayder, quien comunicó el aislamiento de C. difficile de las heces de niños menores de un año de edad. No hubo nuevos informes de aislamiento hasta 1962 cuando Louis de S. Smith y E.O. King, reportaron la presencia de C. difficile en varios tipos de heridas infectadas. Estos investigadores consideraron que el organismo era un saprofito inofensivo en el humano sano, bien porque no produjera su típica toxina o porque ordinariamente, el organismo humano no era susceptible a la potencial acción letal de la toxina. En la segunda edición de su libro Pathogenic Anaerobic Bacteria, publicado en 1975, el Dr. Smith puntualizó: "Aún cuando este organismo ha sido aislado en una variedad de condiciones clínicas del humano, no se puede establecer con certeza que sea patógeno para el hombre". A lo largo de los años, el Laboratorio de Referencia del CDC ha identificado C. difficile en diferentes clases de material humano pero, hasta hace poco, el organismo no había sido asociado con alguna enfermedad en particular y no aparecía como un patógeno de importancia. Recientemente, S. Hafiz y sus colaboradores comunicaron en Inglaterra el aislamiento de C. difficile del tracto urogenital de la mayoría de los hombres y mujeres que asistían a una clínica de venereología y en cambio, en muy posos pacientes atendidos en los servicios de planeación familiar o en clínicas urológicas. 
V. R. DOWELL, JR.

RESULTADOS DE LAS EXPERIENCIAS CON CULTIVOS TISULARES

$Y$ LA TOXINA DE C. DIFFICILE

\begin{tabular}{|c|c|c|c|c|c|c|c|}
\hline \multirow{3}{*}{ Especimen } & \multicolumn{7}{|c|}{ Tratamientos antes de la inoculación de los cultivos celulares } \\
\hline & \multirow{2}{*}{ Ne } & \multirow{2}{*}{$\begin{array}{c}\text { Calor } \\
\left(56^{\circ} \mathrm{C} \times 30 \mathrm{~min}\right)\end{array}$} & \multicolumn{5}{|c|}{ Incubación con Antiroxina de: } \\
\hline & & & C. hisfolyticum & C. perfíingens & C. novyi & C. septicum & C. sordellii \\
\hline $\begin{array}{c}\text { Filtrado de heces del } \\
\text { paciente }\end{array}$ & $\oplus$ & $\Theta$ & $\oplus$ & $\oplus$ & $\oplus$ & $\oplus$ & $\Theta$ \\
\hline $\begin{array}{l}\text { Filtrado de C diffici- } \\
\text { le aislada del paciente. }\end{array}$ & $\oplus$ & $\Theta$ & $\oplus$ & $\oplus$ & $\oplus$ & $\oplus$ & $\Theta$ \\
\hline $\begin{array}{l}\text { Filtrado del cultivo } \\
\text { de referencia de C. di } \\
\text { fficile. }\end{array}$ & $\oplus$ & $\Theta$ & $\oplus$ & $\oplus$ & $\oplus$ & $\oplus$ & $\Theta$ \\
\hline $\begin{array}{l}\text { Filtrado de medio de } \\
\text { cultivo no inoculado } \\
\text { (Control) }\end{array}$ & $\Theta$ & & & & & & \\
\hline $\begin{array}{l}\text { Antitoxina monovalen } \\
\text { te de Clostridium. } \\
\text { (Control) }\end{array}$ & $\Theta$ & & & & & & \\
\hline
\end{tabular}

$($ Presencia de citotoxicidad

$\Theta \quad$ Citotoxicidad ausente

Datos de W.L. George y otros.

La presencio je la toxina de $C$ difficile en las heces de pacientes con colitis seudomembranosa se confirmu for el efecto citopitico de los filtrados fecales en los cultivos celulares. La toxina es neutralizada por el calor y por la antitoxina de $C$. sordellii pero no pur las antitoxinas de otras Clostridia.

Sus evidencias preliminares indicaron que los gérmenes aislados en la clínica de venereología eran patógenos y toxigénicos, no así los aislados de los otros pacientes. Aún cuando no puedieron dilucidar si $C$. difficile representaba a un oportunista o era un agente infectante primario en los pacientes de la clínica de venereología, sí llamaron la atención sobre el uso de la tetraciclina en el tratamiento de la enfermedad gonocócica y las uretritis no específicas.

Hasta la fecha la evidencia que se tiene permite sugerir que el C. difficile forma parte de la flora normal del intestino humano. Gracias a que se le ha encontrado consistentemente en el niño y raramente en el adulto, es de suponer que sea controlado, por así decirlo, por la flora intestinal adquirida durante el crecimiento y el desarrollo. Si produce enterotoxina en el tracto intestinal humano sano, seguramente su cantidad sea mínima como para producir enfermedad, pero posiblemente, cuando la flora intestinal se perturba, el organismo prolifera. Para que el organismo crezca frente a la terapia por antibióticos sería de esperarse que fuera resistente a ellos; paradójicamente, algunas de las cepas de C. difficile aisladas de pacientes con colitis asociada con los antibióticos, han resultado susceptibles a las pruebas in vitro practicadas a los mismos antibióticos que reciben los pacientes. El C. difficile es susceptible casi a todos los antibióticos implicados en la colitis seudomembranosa, incluyendo la clindamicina, la lincomicina, la tetraciclina y las penicilinas semisintéticas. Una posible explicación para este curioso hallazgo la constituye el hecho de que los clostridia esporulan y su esporulación es estimulada en el intestino. El C. perfringens por ejemplo, esporula en el intestino si se ingiere su forma vegetativa. Si el esporo del C. difficile es resistente a los antibióticos y puede sobrevivir a pesar de la susceptibilidad de la forma 
vegetativa, reproduce la forma vegetativa una vez el antibiótico es retirado; en un ambiente post-antibiótico, libre de sus competidores biológicos, el gérmen tiene oportunidad de proliferar. Otra posibilidad sería la de que otros microorganismos, resistentes al antibiótico usado, protegieran al C. difficile en el tracto intestinal.

En los estudios hechos cultivando C. difficile se ha visto que la producción de toxina alcanza un máximo entre el cuarto y el sexto día de incubación, lo cual concuerda con el concepto de una toxina protoplásmica que es liberada con la autolisis de la forma vegetativa del gérmen. Tanto el $C$. botulinum como el C. tetani producen este tipo de toxina, que se libera de tal manera y se presume entonces que ello ocurra con la toxina de C. difficile. En la actualidad, la confirmación de la presencia de la toxina del C. difficile se hace en el laboratorio, tratanto los filtrados de las heces de los pacientes. La técnica más eficiente es la de las inoculaciones en cultivos tisulares, aplicada según previa descripción. No obstante haber empleado para el caso varios tipos de células, tales como WI-38, Hela, Y-1 adrenales y C $\mathrm{V}-1$, hemos encontrado la mayor sensibilidad al efecto citopático de la toxina utilizando cultivos de fibrobastos de prepucio humano en una sola capa.* En el CDC hemos estudiado métodos para el aislamiento del organismo de las muestras fecales y para la estandarización de técnicas que permitan demostrar la toxina en los filtrados de heces y en los líquidos de cultivo. Aún cuando ello no sea necesario para confirmar el diagnóstico, el trabajo pretende desarrollar un procedimiento confirmatorio sencillo, que pueda ser usado por el departamento de Salud del Estado y por el laboratorio hospitalario. Hasta el momento no es sencillo reproducir con seguridad las técnicas in vitro conocidas para aislar el C. difficile o para demostrar la presencia de la toxina en los especímenes clínicos o en los cultivos.

El procedimiento corriente para el aislamiento de C. difficile emplea el alcohol

\footnotetext{
* Por información personal del autor en la actualidad solo se utiliza fibroblastos humanos de pulmón.
}

absoluto o el calor para destruír las formas vegetativas (no los esporos) que puedan encontrarse en las materias fecales del paciente; los esporos que quedan se transfieren luego a medios adecuados para el aislamiento y se incuban en anaerobiosis. A partir del medio de cultivo se prepara un filtrado que se titula cuantitativamente para su toxicidad, por medio de cultivos tisulares y por inyección intraperitoneal de $0,5 \mathrm{ml}$. del material diluído en el ratón. Hemos desarrollado un medio líquido que consistentemente da una buena producción de toxina del C. difficile y esperamos desarrollar una prueba estandarizada para esta enterotoxina.

Quedan una serie de incognitas para resolver acerca de C. difficile. Por ejemplo, no se sabe si todas las cepas producen enterotixina o solamente algunas de ellas lo hacen; además no está clara la relación

\section{SUSCEPTIBILIDAD DE I4 CEPAS DE C. DIFFICILE A I4 ANTIMICROBIANOS}

\begin{tabular}{|l|c|c|c|}
\hline \multicolumn{1}{|c|}{ AGENTE } & $\begin{array}{c}\text { CMI }(\mu \mathrm{g}) \\
\text { MENOR }\end{array}$ & $\begin{array}{c}\text { CMI }(\mu \mathrm{g}) \\
\text { MAYOR }\end{array}$ & $\begin{array}{c}\text { CMI }(\mu \mathrm{g}) \\
\text { PROMEDIO }\end{array}$ \\
\hline Ampicilina & $<1$ & $<1$ & $<1$ \\
Bacitracina & 16 & 32 & 22,9 \\
Cefoxitina & 32 & 128 & 102,9 \\
Cefalotina & 32 & 64 & 38,9 \\
Cloranfenicol & 4 & 32 & 8,3 \\
Clindamicina & 2 & $>1.024$ & 91,6 \\
Cicloserina & $>1.024$ & $>1.024$ & $>1.024$ \\
Eritromicina & $<1$ & 1.024 & 147,1 \\
Lincomicina & 16 & $>1.024$ & 169,1 \\
Metronidasol & $<1$ & $<1$ & $<1$ \\
Penicilina & $<1$ & 4 & 2,8 \\
Rifampin & $<1$ & $<1$ & $<1$ \\
Tetraciclina & $<1$ & 64 & 32,5 \\
Vancomicina & $<1$ & $<1$ & $<1$ \\
\hline
\end{tabular}

+ Unidades $/ \mathrm{ml}$

Basado en datos de George. WL ef al: Zoxigenicidad y sus ceptibilidad antimicrobial de Clostridium difficile. CURR. MICROBIOL. $1.978 ; 1: 55-58$ 
entre el C. difficiley el C. sordellii. Cuando se descubrió que era una enterotixina la responsable de la colitis asociada con los antibióticos, se pensó inicialmente que ésta era producida por C. sordellii, por cuanto su antitoxina y ninguna de las de otros clostridia neutralizaba la toxina. Ocurre, sin embargo, que la antitoxina de C. sordellii empleada era una mezcla de antitoxinas de clostridia producida varios años atrás, almacenada en la oficina de Biológicos de la "Food and Drug Administration" y es muy poca la información que se tiene acerca de la cepa de C. sordellii empleada en su producción. Por consiguiente, se requiere una buena cantidad de trabajo básico para estudiar la fisiología y las relaciones antigénicas de C. difficile y C. sordellii. A pesar de que los estudios bacteriológicos han señalado que solo un $2 \%$ de los adultos sanos son portadores de C. difficile en sus heces, es posible que el organismo esté más diseminado. La falla en el hallazgo del gérmen puede estar más en la carencia de medios selectivos apropiados para su aislamiento que en la rareza de éste en sí.

El riesgo de desarrollar colitis relacionadas con $C$. difficile por tratamiento con antibiótico parece ser mayor para la población muy jóven o anciana y para los pacientes hospitalizados o que padecen enfermedades serias.

Los síntomas de la colitis seudomembranosa suelen aparecer hacia el séptimo día de haberse iniciado la terapia con antibióticos. Posiblemente el tratamiento más efectivo sea vancomicina oral, la cual debe continuarse por siete a diez días a fin de prevenir recaídas.

\section{BIBLIOGRAFIA}

1. Tedesco Fj, Satanley RJ, Alpers DH. Diagnostic features of clindamycin associated pseudomembranous colitis. N. Engl. J. Med. 1974; 15: 841 .

2. Kappas A et al. Diagnosis of pseudomembranous colitis. Br. Med. J. 1978; 1:675.

3. Rifkin Gd, Fekety FR, Silva J. JR, Sanck EB. Antibiotic induced colitis: implication of a toxin neutralizad by Clostridium sordellii antitoxin. Lancet 1977; II : 1. 103.

4. Larson HE, Price AB. Pseudomembranous colitis : presence of clostridial toxin. Lancet 1977; II : 1312.

5. Bartlett JG et al. Antibiotic-associated pseudomembranous colitis due to toxin producing clostridia. N. Engl, J. Med. 1978; 87: 531 .

6. George Wl, Sutter VL, Finegold SM. Toxigenicity and antimicrobial susceptibility of Clostridium difficile, a cause of antimicrobial agent associated colitis. Curr. Microbiol. 1978; 1: 55 .

7. George WL, Sutter VT, Finegold SM. Antimicrobial agent induced diarrhea. J. infect. Dis. $1977 ; 136$ : 822 .

8. Feketey R. Antibiotic-associated pseudomembranous colitis. Clinical Microbiology Newsletter, Preview Issue, Blazevic $\mathrm{Dj}$, McCarty LR, Morello JA (Eds), GK Hall, Boston. 1978.

9. Hummel RP, Altemeier WA, Hill Ed. Iatrogenic staphylococcal enterocolotis. Ann. Surg. 1964; 160: 551 .

10. Tedesco FR et al. Oral Vancomycin for antibiotic -associated pseudomembranous colitis. Lancet 1978; II : 226 . 